\title{
Hybridizing Surrogate Techniques, Rough Sets and Evolutionary Algorithms to Efficiently Solve Multi-Objective Optimization Problems
}

\author{
Luis V. Santana-Quintero \\ CINVESTAV-IPN \\ Computer Science \\ Department \\ MÉXICO \\ Ivspenny@ hotmail.com
}

\author{
Carlos A. Coello Coello \\ CINVESTAV-IPN \\ Computer Science \\ Department \\ MÉXICO \\ ccoello@cs.cinvestav.mx
}

\author{
Alfredo $\mathrm{G}$. \\ Hernández-Díaz \\ Pablo de Olavide University \\ Dept. of Quantitative Methods \\ Seville, Spain \\ agarher@upo.es
}

\begin{abstract}
${ }^{1}$ This paper presents an approach in which a multi-objective evolutionary algorithm (MOEA) is coupled to a surrogate method in order to explore the search space in an efficient manner. A small comparative study among three surrogate methods is conducted: an artificial neural network (ANN), a radial basis function ( $\mathrm{RBF}$ ) and a support vector machine (SVM). The winner in this comparative study was the SVM. However, our results indicated that the spread of solutions achieved by our surrogate-based MOEA was poor. Thus, we decided to introduce a second phase to the algorithm in which it is hybridized with the rough sets in order to improve the spread of solutions and help to reach the true Pareto front. We show that our proposed hybrid approach only requires 2,000 fitness function evaluations in order to solve test problems with up to 30 decision variables.
\end{abstract}

Categories and Subject Descriptors: G.1.2 Numerical Analysis: Special function approximations G.1.6 Numerical Analysis: Unconstrained optimization

General Terms: Algorithms, Performance

Keywords: Multi-objective optimization, surrogates, hybrid algorithms

\section{INTRODUCTION}

In recent years, the statement and solution of multi-objective optimization problems has become very common in a wide variety of disciplines in which computational efficiency is a critical issue. However, multi-objective evolutionary algorithms (MOEAs) are normally expensive in the sense that they require a high number of objective function evaluations (e.g., in aeronautical engineering). Through interpolation, extrapolation and/or integration, surrogate models can be

\footnotetext{
${ }^{1}$ The second author acknowledges support from project CONACyT number: 45683-Y
}

Copyright is held by the author/owner(s). GECCO'08, July 12-16, 2008, Atlanta, Georgia, USA. ACM 978-1-60558-130-9/08/07. used to address complex problems involving experimental design, system analysis and prediction. There exist a variety of techniques for constructing surrogate models [10]. Currently, there exist several evolutionary algorithms that use a meta-model (surrogate) to approximate the real fitness function (see for example $[6,7,12]$ ).

\section{OUR PROPOSED APPROACH}

Our proposed approach is divided in two different phases, each of which consumes a fixed number of fitness function evaluations. In the first phase, our surrogate-based MOEA is applied for 600 fitness function evaluations (this value was empirically derived). In order to determine the most appropriate surrogate method to be adopted, we performed a small comparative study. Although the surrogate-based MOEA can produce reasonably good approximations of the Pareto front, the distribution of solutions is normally poor. This motivated a second phase for our approach, in which rough sets are applied as a local search engine during 1,400 fitness evaluations in order to improve the solutions produced at the previous phase.

Phase 1: Surrogate-based MOEA. The surrogate model adopted in this work is shown in Figure 1. A multiobjective particle swarm optimizer (MOPSO) is adopted to optimize the approximate model generated by the different surrogates (artificial neural networks (ANNs)[1], radial basis functions (RBFs), and support vector machines (SVMs) [9]). Our MOPSO maintains two populations: the main one (which is used to select the leaders), and a second population that retains some solutions that were dominated by the global nondominated solutions that are needed for the second phase. Both populations use the pat-dominance grid proposed in [5] to maintain diversity.

Phase 2: Rough Sets in Multi-Objective Optimization. Rough Sets theory is a mathematical approach to deal with imperfect knowledge originally proposed by Pawlak [8]. We will try to approximate the Pareto front using a Rough Sets grid. In order to do this, we will use an initial approximation of the Pareto front (provided by the surrogate algorithm) and will implement a grid in order to get more information about the front that will let us improve this initial approximation. To create this grid, we use $N$ feasible points divided in two sets: the nondominated points $(E S)$ and the dominated ones $(D S)$. Using these two sets we want to create a grid to describe the set $E S$ in order to intensify the search on it (see [4] for further details). 


\begin{tabular}{|c|c|c|c|c|c|c|c|c|c|c|c|c|c|c|}
\hline ZDT1 & ANN & $\overline{R B F}$ & SVM & Mean & ZDT2 & ANN & RBF & SVM & Mean & ZDT3 & ANN & RBF & SVM & Mean \\
\hline ANN & - & 0.1033 & 0.9561 & 0.5297 & ANN & - & 0.2667 & \multirow{2}{*}{1.0} & \multirow{2}{*}{0.6333} & ANN & \multirow{2}{*}{$\begin{array}{c}- \\
0.8584\end{array}$} & 0.1 & 0.9165 & \multirow{2}{*}{$\begin{array}{l}0.5082 \\
0.8867\end{array}$} \\
\hline RBF & 0.8581 & $\begin{array}{c}0.1000 \\
-\end{array}$ & 0.9256 & 0.8918 & $\mathrm{RBF}$ & 0.75 & \multirow{2}{*}{$\begin{array}{c}- \\
0.0\end{array}$} & & & RBF & & - & 0.9150 & \\
\hline SVM & 0.0 & 0.0 & - & 0.0 & SVM & 0.0 & & - & 0.0 & SVM & 0.0 & 0.0 & - & 0.0 \\
\hline & & & ZDT4 & ANN & $\overline{\mathrm{BF}}$ & $\mathrm{M}$ & Mean & ZDT6 & ANN & $\mathrm{RBF}$ & SVM & Mean & & \\
\hline & & & ANN & - & 3717 & 83 & 0.7508 & ANN & - & 0.4582 & 0.7864 & 0.6223 & & \\
\hline & & & $\mathrm{RBF}$ & 0.2824 & - & 774 & 0.5299 & $\mathrm{RBF}$ & 0.2696 & - & 0.7280 & 0.4988 & & \\
\hline & & & SVM & 0.0250 & .1 & - & 0.0625 & SVM & 0.0 & 0.0 & - & 0.0 & & \\
\hline
\end{tabular}

Table 1: Results of Set Coverage (SC) for the ZDT test problems (600 evaluations).

\begin{tabular}{|c|c|c|c|c|c|c|c|c|c|c|c|c|}
\hline & \multicolumn{4}{|c|}{ Set Coverage } & \multicolumn{4}{|c|}{ IGD } & \multicolumn{4}{|c|}{ Spread } \\
\hline Function & \multicolumn{2}{|c|}{ SVM+RS } & \multicolumn{2}{|c|}{ NSGA-II } & \multicolumn{2}{|c|}{ SVM+RS } & \multicolumn{2}{|c|}{ NSGA-II } & \multicolumn{2}{|c|}{ SVM+RS } & \multicolumn{2}{|c|}{ NSGA-II } \\
\hline & Mean & $\sigma$ & Mean & $\sigma$ & Mean & $\sigma$ & Mean & $\sigma$ & Mean & $\sigma$ & Mean & $\sigma$ \\
\hline ZDT1 & 0.0725 & 0.1676 & 0.8755 & 0.1785 & 0.0061 & 0.0046 & 0.0168 & 0.0021 & 0.8308 & 0.1987 & 0.8194 & 0.0324 \\
\hline ZDT2 & 0.0000 & 0.0000 & 0.9690 & 0.0505 & 0.0026 & 0.0027 & 0.0382 & 0.0062 & 0.5684 & 0.2338 & 0.9711 & 0.0696 \\
\hline ZDT3 & 0.0372 & 0.0668 & 0.6453 & 0.3343 & 0.0145 & 0.0071 & 0.1190 & 0.0113 & 0.8684 & 0.0943 & 0.9475 & 0.0296 \\
\hline ZDT4 & 0.7937 & 0.2512 & 0.0167 & 0.0500 & 0.7397 & 0.1554 & 0.1511 & 0.0405 & 0.9572 & 0.0411 & 1.0795 & 0.1026 \\
\hline ZDT6 & 0.0000 & 0.0000 & 0.9208 & 0.1886 & 0.0135 & 0.0025 & 0.0548 & 0.0092 & 1.1000 & 0.1297 & 0.9588 & 0.0274 \\
\hline
\end{tabular}

Table 2: Results of SVM+RS and the NSGA-II for the ZDT test problems (2000 evaluations).

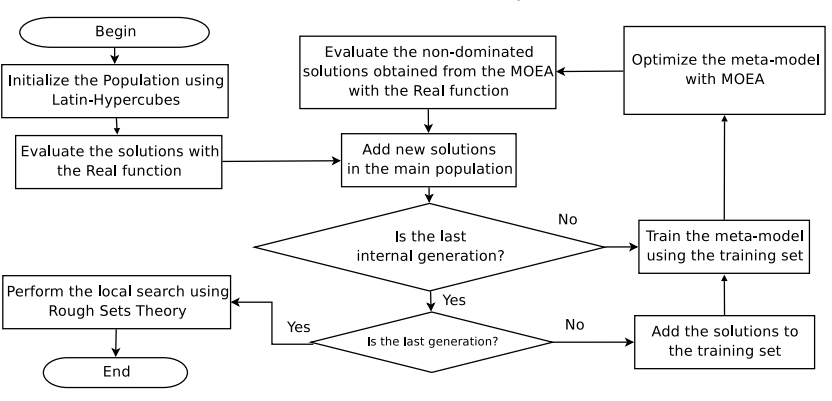

Figure 1: Surrogate model adopted in this paper.

\section{ANALYSIS OF RESULTS}

For our experiments, we adopted the ZDT test problems [13]. Three performance measures were adopted in order to allow a quantitative assessment of our results: (1) Inverted Generational Distance (IGD) [11]; (2) Two Set Coverage (SC) [13]; and (3) Spread (S)[2]. For each test problem, 10 independent runs were performed.

In this study, we perform 600 real function evaluations using different surrogate methods: ANNs, RBFs and SVMs. The results reported in Table 1 correspond to the SC metric. From the results, we can conclude that the SVM approach is the one that shows the best overall performance in these particular multi-dimensional test functions. So, we decided to choose this approach to hybridize it with the Rough Sets.

We decided to compare our hybrid (using SVM and RS) with an algorithm representative of the state of the art (NSGA-II) [3]. The parameters used by the NSGA-II are: $c_{r}=0.9, m_{r}=1 / n, \eta_{c}=15, \eta_{m}=20$, population size $=$ 52 and maximum number of generations $=39$. Making the NSGA-II to perform 2,028 fitness function evaluations.

The results are reported in Table 2 for the three performance measures (SC, IGD and S) performing the same number of evaluations for SVM+RS and NSGA-II (allowing a fair comparison between both approaches). In the ZDT test problems, our approach produced the best results with respect to SC and IGD in all cases except for ZDT4. The poor performance in ZDT4 might be attributed to the bad scalability presented by both approaches.

\section{CONCLUSIONS AND FUTURE WORK}

We have presented an initial study of surrogate methods to solve bi-objective problems. Three different methods were used: ANNs, RBFs and SVMs, all of them in their regression form to approximate the functions using supervised learning. From this study, we concluded that the SVMs were the most appropriate model for dealing with the kind of problems of our interest, although it is unable to produce a good spread of solutions. Thus, we included a local search procedure based on rough sets theory to intensify the search around the solutions obtained by the SVMs. This hybrid algorithm provides competitive results in most of the test problems adopted. In future work, we are interested in refining the interaction mechanism between the surrogate method and the MOEA, and use an Ensemble Method to help the surrogate models get a better approximation of the real functions.

\section{REFERENCES}

[1] C. M. Bishop. Neural Networks for Pattern Recognition. Oxford University Press, UK, 1995.

[2] K. Deb. Multi-Objective Optimization using Evolutionary Algorithms. John Wiley \& Sons, Chichester, UK, 2001. ISBN 0-471-87339-X.

[3] K. Deb, A. Pratap, S. Agarwal, and T. Meyarivan. A Fast and Elitist Multiobjective Genetic Algorithm: NSGA-II. IEEE Transactions on Evolutionary Computation, 6(2):182-197, April 2002.

[4] A. G. Hernández-Díaz, L. V. Santana-Quintero, C. A. C. Coello, R. Caballero, and J. Molina. Improving multi-objective evolutionary algorithms by using rough sets. In Knowledge-Driven Computing. Springer, 2008.

[5] A. G. Hernández-Díaz, L. V. Santana-Quintero, C. A. Coello Coello, and J. Molina. Pareto-adaptive $\epsilon$-dominance. Evolutionary Computation, 15(4):493-517, Winter 2007.

[6] Y. Jin. A comprehensive survey of fitness approximation in evolutionary computation. Soft Computing, 9(1):3-12, 2005.

[7] J. Knowles. ParEGO: A hybrid algorithm with on-line landscape approximation for expensive multiobjective optimization problems. IEEE Transactions on Evolutionary Computation, 10(1):50-66, January 2006.

[8] Z. Pawlak. Rough sets. International Journal of Computer and Information Sciences, 11(1):341-356, Summer 1982.

[9] A. J. Smola and B. Schölkopf. A tutorial on support vector regression. Technical report, NeuroCOLT, September 2003.

[10] V. N. Vapnik. Statistical Learning Theory. Wiley, 1998.

[11] D. A. V. Veldhuizen. Multiobjective Evolutionary Algorithms: Classifications, Analyses, and New Innovations. PhD thesis, Department of Electrical and Computer Engineering. Air Force Institute of Technology, Ohio, May 1999.

[12] I. Voutchkov and A. Keane. Multiobjective Optimization using Surrogates. In I. Parmee, editor, Adaptive Computing in Design and Manufacture. Proceedings of the Seventh International Conference, pages 167-175, 2006.

[13] E. Zitzler, K. Deb, and L. Thiele. Comparison of Multiobjective Evolutionary Algorithms: Empirical Results. Evolutionary Computation, 8(2):173-195, Summer 2000. 